\title{
On the Stabilization for Laminated Beam with Infinite Memory and Different Speeds of Wave Propagation
}

\author{
Gang Li and Xiangyu Kong \\ College of Mathematics and Statistics, Nanjing University of Information Science and Technology, \\ Nanjing 210044, China. E-mail: m15651559885@163.com
}

In this work, we consider a one-dimensional laminated beam in the case of non-equal wave speeds with only one infinite memory on the effective rotation angle. In this case, we establish the general decay result for the energy of solution without any kind of internal or boundary control. The main result is obtained by applying the method used in Guesmia et al. (Electron. J. Differential Equations 193: 1-45, 2012) and the second-order energy.

Keywords: general stability, laminated beam, infinite memory, multiplier technique, energy method.

AMS Subject Classification (2000): 34B05, 35L05, 93C20, 93D20.

\section{Introduction}

In this paper, we are concerned the following initial boundary value problem

$$
\begin{cases}\rho \varphi_{t t}+G\left(\psi-\varphi_{x}\right)_{x}=0, & (x, t) \in(0,1) \times(0,+\infty), \\ I_{\rho}(3 w-\psi)_{t t}-G\left(\psi-\varphi_{x}\right)-D(3 w-\psi)_{x x} & \\ \quad+\int_{0}^{+\infty} g(s)(3 w-\psi)_{x x}(x, t-s) \mathrm{d} s=0, & (x, t) \in(0,1) \times(0,+\infty), \\ I_{\rho} w_{t t}+G\left(\psi-\varphi_{x}\right)+\frac{4}{3} \gamma w+\frac{4}{3} \beta w_{t}-D w_{x x}=0, & (x, t) \in(0,1) \times(0,+\infty), \\ \varphi(x, 0)=\varphi_{0}(x), \psi(x,-t)=\psi_{0}(x, t), w(x,-t)=w_{0}(x, t), & (x, t) \in[0,1] \times(0,+\infty), \\ \varphi_{t}(x, 0)=\varphi_{1}(x), \psi_{t}(x, 0)=\psi_{1}(x), w_{t}(x, 0)=w_{1}(x), & x \in[0,1], \\ \varphi_{x}(0, t)=\varphi_{x}(1, t)=\psi(0, t)=\psi(1, t)=w(0, t)=w(1, t)=0, t \in[0,+\infty),\end{cases}
$$

where $g: \mathbb{R}^{+} \rightarrow \mathbb{R}^{+}$is a given function and $\rho, G, I_{\rho}, D, \gamma, \beta$ are positive constants. The infinite integral term in (1.1) represents the infinite memory.

The model for this structure has been derived and treated in [7]. The authors considered the following system

$$
\begin{cases}\rho \varphi_{t t}+G\left(\psi-\varphi_{x}\right)_{x}=0, & (x, t) \in(0,1) \times(0,+\infty), \\ I_{\rho}(3 w-\psi)_{t t}-G\left(\psi-\varphi_{x}\right)-D(3 w-\psi)_{x x}=0, & (x, t) \in(0,1) \times(0,+\infty), \\ 3 I_{\rho} w_{t t}+3 G\left(\psi-\varphi_{x}\right)+4 \gamma w+4 \beta w_{t}-3 D w_{x x}=0, & (x, t) \in(0,1) \times(0,+\infty)\end{cases}
$$

with the initial data and the cantilever boundary conditions. Here $\varphi(x, t)$ denotes the transverse displacement of the beam which departs from its equilibrium position, $\psi(x, t)$ represents the rotation angle, $w(x, t)$ is proportional to the amount of slip along the interface at time $t$ and longitudinal spatial variable $x, 3 w-\psi$ denotes the effective rotation angle and the third equation of 1.2 describes the dynamics of the slip. The coefficients $\rho, G, I_{\rho}, D, \gamma, \beta>0$ denote the density 
of the beams, the shear stiffness, the mass moment of inertia, the flexural rigidity, the adhesive stiffness of the beams, and the adhesive damping parameter, respectively. For the stability of the laminated beams with finite memory, Lo and Tatar [10] studied a laminated beam with a finite memory acting on the effective rotation angle. The authors showed that for viscoelastic material there is no need for any kind of internal or boundary control to stabilize exponentially the system of laminated beams with interfacial slip. For more papers concerning the laminated beam, we refer to [11, 14, 16].

Moreover, it is well known that when $w \equiv 0$, we recover the standard Timoshenko system. Up to now, many people have been interested in the question of stability of Timoshenko systems with infinite memory and different speeds of wave propagation. Guesmia et al. [6] considered a vibrating system of Timoshenko type in a one-dimensional bounded domain with an infinite history acting in the equation of the rotation angle of the form

$$
\left\{\begin{array}{lr}
\rho_{1} \varphi_{t t}-k_{1}\left(\varphi_{x}+\psi\right)_{x}=0, & (x, t) \in(0, L) \times(0,+\infty), \\
\rho_{2} \psi_{t t}-k_{2} \psi_{x x}+k_{1}\left(\varphi_{x}+\psi\right)+\int_{0}^{+\infty} g(s) \psi_{x x}(x, t-s) \mathrm{d} s=0, & (x, t) \in(0, L) \times(0,+\infty) .
\end{array}\right.
$$

The authors proved a general decay of the solution for the case of equal-speed wave propagation as well as for the nonequal-speed case. For more papers related to the Timoshenko system with memory or different speeds of wave propagation, we refer the reader to [3, 4, 5, 8, 12, 15.

In this paper, for a wide class of relaxation function, we intend to study the general decay rate of the solutions for problem (1.1) under non-equal wave speeds. For this purpose, we have two key points in the proofs. On the one hand, to deal with the infinite memory, we introduce the method used in [6]. On the other hand, to estimate the non-equal wave speeds term in (3.21), we use the second-order energy. This is motivated by Guesmia et al.'s work [6], in which a linear Timoshenko system with infinite memory was studied and a general decay result was established for the case of equal-speed wave propagation and the opposite one.

The remaining part of this paper is organized as follows. In section 2, we present some hypotheses needed for our work and state the main results. In section 3, we prove the general decay result of problem (1.1) for the relaxation function $g$ satisfying assumption (G1) and (G2).

\section{Preliminaries and main results}

In this section, we begin with some materials and known results for problem (1.1). For the relaxation function $g$, we have the following assumptions:

(G1) $g: \mathbb{R}^{+} \rightarrow \mathbb{R}^{+}$is a nonincreasing differentiable function such that

$$
g(0)>0, \quad D-\int_{0}^{+\infty} g(s) \mathrm{d} s=D-g_{0}=l>0 .
$$

(G2) There exists an increasing strictly convex function $G: \mathbb{R}^{+} \rightarrow \mathbb{R}^{+}$of class $C^{1}\left(\mathbb{R}^{+}\right) \cap$ $C^{2}(0,+\infty)$ satisfying

$$
G(0)=G^{\prime}(0)=0 \quad \text { and } \quad \lim _{t \rightarrow+\infty} G^{\prime}(t)=+\infty
$$

such that

$$
\int_{0}^{+\infty} \frac{g(s)}{G^{-1}\left(-g^{\prime}(s)\right)} \mathrm{d} s+\sup _{s \in \mathbb{R}^{+}} \frac{g(s)}{G^{-1}\left(-g^{\prime}(s)\right)}<+\infty
$$


Following the ideal of [2], we first set

$$
\eta(x, t, s)=(3 w-\psi)(x, t)-(3 w-\psi)(x, t-s),(x, t, s) \in(0,1) \times(0,+\infty) \times(0,+\infty) .
$$

Then, we have

$$
\eta_{t}+\eta_{s}-(3 w-\psi)_{t}=0,(x, t, s) \in(0,1) \times(0,+\infty) \times(0,+\infty) .
$$

Therefore, problem (1.1) is equivalent to

$$
\begin{cases}\rho \varphi_{t t}+G\left(\psi-\varphi_{x}\right)_{x}=0, & (x, t) \in(0,1) \times(0,+\infty), \\ I_{\rho}(3 w-\psi)_{t t}-G\left(\psi-\varphi_{x}\right)-l(3 w-\psi)_{x x} & \\ \quad-\int_{0}^{+\infty} g(s) \eta_{x x}(x, s) \mathrm{d} s=0, & (x, t, s) \in(0,1) \times(0,+\infty) \times(0,+\infty), \\ I_{\rho} w_{t t}+G\left(\psi-\varphi_{x}\right)+\frac{4}{3} \gamma w+\frac{4}{3} \beta w_{t}-D w_{x x}=0, & (x, t) \in(0,1) \times(0,+\infty), \\ \eta_{t}+\eta_{s}-(3 w-\psi)_{t}=0, & (x, t, s) \in(0,1) \times(0,+\infty) \times(0,+\infty)\end{cases}
$$

with the initial data and boundary conditions

$$
\begin{cases}\varphi(x, 0)=\varphi_{0}(x), \psi(x, 0)=\psi_{0}(x), w(x, 0)=w_{0}(x), & x \in[0,1], \\ \varphi_{t}(x, 0)=\varphi_{1}(x), \psi_{t}(x, 0)=\psi_{1}(x), w_{t}(x, 0)=w_{1}(x), & x \in[0,1], \\ \varphi_{x}(0, t)=\varphi_{x}(1, t)=\psi(0, t)=\psi(1, t)=w(0, t)=w(1, t)=0, & t \in[0,+\infty), \\ \eta(0, t, s)=\eta(1, t, s)=0, & (t, s) \in[0,+\infty) \times[0,+\infty), \\ \eta(x, t, 0)=0, & (x, t) \in[0,1] \times(0,+\infty), \\ \eta(x, 0, s)=\eta_{0}(x, s), & (x, s) \in[0,1] \times(0,+\infty) .\end{cases}
$$

Next, let

$$
U=\left(\varphi, 3 w-\psi, w, \varphi_{t}, 3 w_{t}-\psi_{t}, w_{t}, \eta\right)^{T}
$$

and

$$
U^{0}(x)=\left(\varphi_{0}(x), 3 w_{0}(x)-\psi_{0}(x), w_{0}(x), \varphi_{1}(x), 3 w_{1}(x)-\psi_{1}(x), w_{1}(x), \eta_{0}(x, s)\right)^{T} .
$$

Hence, problem (2.5)-2.6) is equivalent to the following abstract Cauchy problem:

$$
\left\{\begin{array}{l}
\partial_{t} U=\mathscr{A} U, \\
U(x, 0)=U^{0}(x),
\end{array}\right.
$$


where $\mathscr{A}$ is a linear operator

$$
\mathscr{A} U=\left(\begin{array}{c}
\varphi_{t} \\
3 w_{t}-\psi_{t} \\
w_{t} \\
-\frac{G}{\rho}\left(\psi-\varphi_{x}\right)_{x} \\
\frac{l}{I_{\rho}}\left(\psi-\varphi_{x}\right)+\frac{l}{I_{\rho}}(3 w-\psi)_{x x}+\frac{1}{I_{\rho}} \int_{0}^{+\infty} g(s) \eta_{x x}(x, s) \mathrm{d} s \\
-\frac{G}{I_{\rho}}\left(\psi_{-} \varphi_{x}\right)-\frac{4 \gamma}{3 I_{\rho}} w-\frac{4 \beta}{3 I_{\rho}} w_{t}+\frac{D}{I_{\rho}} w_{x x} \\
-\eta_{s}+(3 w-\psi)_{t}
\end{array}\right) .
$$

Now, we consider the following spaces:

$$
\begin{gathered}
L_{*}^{2}(0,1)=\left\{\varphi \mid \varphi \in L^{2}(0,1): \int_{0}^{1} \varphi(x) \mathrm{d} x=0\right\}, \quad H_{*}^{1}=H^{1}(0,1) \cap L_{*}^{2}(0,1), \\
H_{*}^{2}=\left\{\varphi \mid \varphi \in H^{2}(0,1): \varphi_{x}(0)=\varphi_{x}(1)=0\right\}
\end{gathered}
$$

and define the functional space of $U$ as follows:

$$
\mathscr{H}=H_{*}^{1}(0,1) \times\left(H_{0}^{1}(0,1)\right)^{2} \times L_{*}^{2}(0,1) \times\left(L^{2}(0,1)\right)^{2} \times L_{g},
$$

where

$$
L_{g}=\left\{\eta \mid \eta: \mathbb{R}^{+} \longrightarrow H_{0}^{1}(0,1), \int_{0}^{1} \int_{0}^{+\infty} g(s) \eta_{x}^{2}(x, s) \mathrm{d} s \mathrm{~d} x<+\infty\right\} .
$$

Then, the domain of $\mathscr{A}$ is defined by

$$
D(\mathscr{A})=H_{*}^{2}(0,1) \cap H_{*}^{1}(0,1) \times\left(H^{2}(0,1) \cap H_{0}^{1}(0,1)\right)^{2} \times H_{*}^{1}(0,1) \times\left(H_{0}^{1}(0,1)\right)^{2} \times \mathscr{L}_{g},
$$

where

$$
\mathscr{L}_{g}=\left\{\eta \mid \eta \in L_{g}, \eta_{s} \in L_{g}, \eta(x, t, 0)=0\right\}
$$

To state our decay result, we introduce the following energy functional:

$$
\begin{aligned}
E(t)= & \frac{1}{2} \int_{0}^{1}\left(\rho \varphi_{t}^{2}+I_{\rho}(3 w-\psi)_{t}^{2}+3 I_{\rho} w_{t}^{2}+G\left(\psi-\varphi_{x}\right)^{2}+l(3 w-\psi)_{x}^{2}+3 D w_{x}^{2}+4 \gamma w^{2}\right) \mathrm{d} x \\
& +\frac{1}{2} \int_{0}^{1} \int_{0}^{+\infty} g(s) \eta_{x}^{2}(x, s) \mathrm{d} s \mathrm{~d} x .
\end{aligned}
$$

Our decay result reads as follows: 
Theorem 2.1 Assume that $(G 1),(G 2)$ and $\frac{\rho}{G} \neq \frac{I_{\rho}}{D}$ hold. For any $U^{0} \in D(\mathscr{A})$ satisfying, for some $M_{0} \geq 0$,

$$
\max \left\{\int_{0}^{1} \eta_{0 x}^{2}(x, s) \mathrm{d} x, \int_{0}^{1} \eta_{0 x s}^{2}(x, s) \mathrm{d} x\right\} \leq M_{0}, \quad \forall s \geq 0 .
$$

Then, there exist positive constants $C, \epsilon_{0}$ such that the energy $E(t)$ associated with problem (1.1) satisfies

$$
E(t) \leq G_{0}^{-1}\left(\frac{C}{t}\right), \quad \forall t \geq 0
$$

where

$$
G_{0}(s)=s G^{\prime}\left(\epsilon_{0} s\right)\left(s \in \mathbb{R}^{+}\right)
$$

\section{General decay of the solution}

In this section, we prove the general decay result as stated in Theorem 2.1. It will be accomplished by constructing a Lyapunov functional $L(t)$ equivalent to $E(t)$.

Before proving our main result, we will state and prove some useful lemmas in advance.

Lemma 3.1 The energy functional $E(t)$ defined by $(2.9)$ satisfies

$$
\frac{d}{d t} E(t)=-4 \beta \int_{0}^{1} w_{t}^{2} \mathrm{~d} x+\frac{1}{2} \int_{0}^{1} \int_{0}^{+\infty} g^{\prime}(s) \eta_{x}^{2}(x, s) \mathrm{d} s \mathrm{~d} x .
$$

Proof. Multiplying the first equation of (2.1) by $\varphi_{t}$ and integrating over $(0,1)$, we have

$$
\frac{1}{2} \frac{d}{d t} \int_{0}^{1} \rho \varphi_{t}^{2} \mathrm{~d} x+\int_{0}^{1} G\left(\psi-\varphi_{x}\right)_{x} \varphi_{t} \mathrm{~d} x=0
$$

Then using integration by parts and the boundary conditions in 2.2 , we obtain

$$
\frac{1}{2} \frac{d}{d t} \int_{0}^{1} \rho \varphi_{t}^{2} \mathrm{~d} x-\int_{0}^{1} G\left(\psi-\varphi_{x}\right) \varphi_{x t} \mathrm{~d} x=0 .
$$

Note that

$$
\begin{aligned}
\int_{0}^{1} G\left(\psi-\varphi_{x}\right) \varphi_{x t} \mathrm{~d} x & =-\int_{0}^{1} G\left(\psi-\varphi_{x}\right)\left(\psi-\varphi_{x}-\psi\right)_{t} \mathrm{~d} x \\
& =-\frac{1}{2} \frac{d}{d t} \int_{0}^{1} G\left(\psi-\varphi_{x}\right)^{2} \mathrm{~d} x+\int_{0}^{1} G\left(\psi-\varphi_{x}\right) \psi_{t} \mathrm{~d} x .
\end{aligned}
$$

Hence, equation $(3.2)$ becomes

$$
\frac{1}{2} \frac{d}{d t} \int_{0}^{1}\left(\rho \varphi_{t}^{2}+G\left(\psi-\varphi_{x}\right)^{2}\right) \mathrm{d} x-\int_{0}^{1} G\left(\psi-\varphi_{x}\right) \psi_{t} \mathrm{~d} x=0 .
$$

Next, multiplying the second equation of 2.1 by $3 w_{t}-\psi_{t}$ and integrating over $(0,1)$, we get

$$
\int_{0}^{1} I_{\rho}(3 w-\psi)_{t t}(3 w-\psi)_{t} \mathrm{~d} x-\int_{0}^{1} G\left(\psi-\varphi_{x}\right)(3 w-\psi)_{t} \mathrm{~d} x
$$




$$
-\int_{0}^{1} l(3 w-\psi)_{x x}(3 w-\psi)_{t} \mathrm{~d} x-\int_{0}^{1} \int_{0}^{+\infty} g(s) \eta_{x x}(x, s) \mathrm{d} s(3 w-\psi)_{t} \mathrm{~d} x=0 .
$$

Then, integrating by parts, using the boundary conditions in 2.2 and $\left(G_{1}\right)$, we arrive at

$$
\begin{gathered}
\frac{1}{2} \frac{d}{d t} \int_{0}^{1}\left(I_{\rho}(3 w-\psi)_{t}^{2}+l(3 w-\psi)_{x}^{2}\right) \mathrm{d} x+\frac{1}{2} \frac{d}{d t} \int_{0}^{1} \int_{0}^{+\infty} g(s) \eta_{x}^{2}(x, s) \mathrm{d} s \mathrm{~d} x \\
-\int_{0}^{1} G\left(\psi-\varphi_{x}\right)(3 w-\psi)_{t} \mathrm{~d} x-\frac{1}{2} \int_{0}^{1} \int_{0}^{+\infty} g^{\prime}(s) \eta_{x}^{2}(x, s) \mathrm{d} s \mathrm{~d} x=0 .
\end{gathered}
$$

Similarly, multiplying the third equation of (2.1) by $3 w_{t}$ and integrating over $(0,1)$, we have

$$
\int_{0}^{1} 3 I_{\rho} w_{t t} w_{t} \mathrm{~d} x+\int_{0}^{1} 3 G\left(\psi-\varphi_{x}\right) w_{t} \mathrm{~d} x+\int_{0}^{1} 4 \gamma w w_{t} \mathrm{~d} x+\int_{0}^{1} 4 \beta w_{t}^{2} \mathrm{~d} x-\int_{0}^{1} 3 D w_{x x} w_{t} \mathrm{~d} x=0 .
$$

Then, integrating by parts and using the boundary conditions in 2.2 , we obtain

$$
\frac{1}{2} \frac{d}{d t} \int_{0}^{1}\left(3 I_{\rho} w_{t}^{2}+4 \gamma w^{2}+3 D w_{x}^{2}\right) \mathrm{d} x+\int_{0}^{1} 3 G\left(\psi-\varphi_{x}\right) w_{t} \mathrm{~d} x+\int_{0}^{1} 4 \beta w_{t}^{2} \mathrm{~d} x=0 .
$$

Finally, adding (3.3)-3.5), the proof is complete.

Next, to construct a Lyapunov functional equivalent to the energy, we will prove several lemmas with the purpose of creating negative counterparts of the terms that appear in the energy.

As in [10], we consider the following functionals:

$$
I_{1}(t)=-\rho \int_{0}^{1} \varphi \varphi_{t} \mathrm{~d} x, \quad I_{2}(t)=I_{\rho} \int_{0}^{1}(3 w-\psi)(3 w-\psi)_{t} \mathrm{~d} x \quad \text { and } \quad I_{3}(t)=I_{\rho} \int_{0}^{1} w w_{t} \mathrm{~d} x .
$$

Then the following result holds:

Lemma 3.2 The functional $I_{1}(t)$ satisfies, for any $\varepsilon_{1}>0$,

$$
I_{1}^{\prime}(t) \leq-\rho \int_{0}^{1} \varphi_{t}^{2} \mathrm{~d} x+\left(G+\varepsilon_{1}\right) \int_{0}^{1}\left(\psi-\varphi_{x}\right)^{2} \mathrm{~d} x+c\left(\varepsilon_{1}\right) \int_{0}^{1}(3 w-\psi)_{x}^{2} \mathrm{~d} x+c\left(\varepsilon_{1}\right) \int_{0}^{1} w_{x}^{2} \mathrm{~d} x
$$

where $c\left(\varepsilon_{1}\right)$ is a positive constant depending on $\varepsilon_{1}$.

Proof. Differentiating $I_{1}(t)$ with respect to $t$, using the first equation of problem (2.1) and integrating by parts, we have

$$
\begin{aligned}
I_{1}^{\prime}(t) & =-\rho \int_{0}^{1} \varphi_{t}^{2} \mathrm{~d} x-G \int_{0}^{1} \varphi_{x}\left(\psi-\varphi_{x}\right) \mathrm{d} x \\
& =-\rho \int_{0}^{1} \varphi_{t}^{2} \mathrm{~d} x+G \int_{0}^{1}\left(\psi-\varphi_{x}\right)\left(\psi-\varphi_{x}\right) \mathrm{d} x-G \int_{0}^{1} \psi\left(\psi-\varphi_{x}\right) \mathrm{d} x .
\end{aligned}
$$

Making use of Young's and Poincaré's inequalities for the last term of this equality, we get,

$$
I_{1}^{\prime}(t) \leq-\rho \int_{0}^{1} \varphi_{t}^{2} \mathrm{~d} x+G \int_{0}^{1}\left(\psi-\varphi_{x}\right)^{2} \mathrm{~d} x+\varepsilon_{1} G \int_{0}^{1}\left(\psi-\varphi_{x}\right)^{2} \mathrm{~d} x+\frac{C_{p} G}{4 \varepsilon_{1}} \int_{0}^{1} \psi_{x}^{2} \mathrm{~d} x,
$$

where $C_{p}$ is the poincaré constant. Note that

$$
\int_{0}^{1} \psi_{x}^{2} \mathrm{~d} x=\int_{0}^{1}(\psi-3 w+3 w)_{x}^{2} \mathrm{~d} x
$$


Then

$$
\begin{aligned}
I_{1}^{\prime}(t) & \leq-\rho \int_{0}^{1} \varphi_{t}^{2} \mathrm{~d} x+\left(G+\varepsilon_{1} G\right) \int_{0}^{1}\left(\psi-\varphi_{x}\right)^{2} \mathrm{~d} x+\frac{C_{p} G}{2 \varepsilon_{1}} \int_{0}^{1}(\psi-3 w)_{x}^{2} \mathrm{~d} x+\frac{9 C_{p} G}{2 \varepsilon_{1}} \int_{0}^{1} w_{x}^{2} \mathrm{~d} x \\
& \leq-\rho \int_{0}^{1} \varphi_{t}^{2} \mathrm{~d} x+\left(G+\varepsilon_{1}\right) \int_{0}^{1}\left(\psi-\varphi_{x}\right)^{2} \mathrm{~d} x+c\left(\varepsilon_{1}\right) \int_{0}^{1}(\psi-3 w)_{x}^{2} \mathrm{~d} x+c\left(\varepsilon_{1}\right) \int_{0}^{1} w_{x}^{2} \mathrm{~d} x .
\end{aligned}
$$

Hence, the assertion follows immediately.

Lemma 3.3 The functional $I_{2}(t)$ satisfies, for any $\varepsilon_{2}>0$,

$$
\begin{aligned}
I_{2}^{\prime}(t) \leq & -\left(l-\varepsilon_{2}\right) \int_{0}^{1}(3 w-\psi)_{x}^{2} \mathrm{~d} x+I_{\rho} \int_{0}^{1}(3 w-\psi)_{t}^{2} \mathrm{~d} x \\
& +c\left(\varepsilon_{2}\right) \int_{0}^{1}\left(\psi-\varphi_{x}\right)^{2} \mathrm{~d} x+c\left(\varepsilon_{2}\right) \int_{0}^{1} \int_{0}^{+\infty} g(s) \eta_{x}^{2}(x, s) \mathrm{d} s \mathrm{~d} x
\end{aligned}
$$

where $c\left(\varepsilon_{2}\right)$ is a positive constant depending on $\varepsilon_{2}$.

Proof. Taking the derivative of $I_{2}(t)$ with respect to $t$, using the second equation of problem (2.1) and integrating by parts, we obtain

$$
\begin{aligned}
I_{2}^{\prime}(t)= & I_{\rho} \int_{0}^{1}(3 w-\psi)_{t}^{2} \mathrm{~d} x-l \int_{0}^{1}(3 w-\psi)_{x}^{2} \mathrm{~d} x \\
& +G \int_{0}^{1}(3 w-\psi)\left(\psi-\varphi_{x}\right) \mathrm{d} x-\int_{0}^{1}(3 w-\psi)_{x} \int_{0}^{+\infty} g(s) \eta_{x}(x, s) \mathrm{d} s \mathrm{~d} x .
\end{aligned}
$$

Then, by using the Young's, Poincaré's and Höider's inequalities for the last two terms of this equality, we have

$$
\begin{aligned}
I_{2}^{\prime}(t) \leq & I_{\rho} \int_{0}^{1}(3 w-\psi)_{t}^{2} \mathrm{~d} x-l \int_{0}^{1}(3 w-\psi)_{x}^{2} \mathrm{~d} x+\varepsilon_{2} C_{p} G \int_{0}^{1}(3 w-\psi)_{x}^{2} \mathrm{~d} x \\
& +\frac{G}{4 \varepsilon_{2}} \int_{0}^{1}\left(\psi-\varphi_{x}\right)^{2} \mathrm{~d} x+\varepsilon_{2} \int_{0}^{1}(3 w-\psi)_{x}^{2} \mathrm{~d} x+\frac{g^{0}}{4 \varepsilon_{2}} \int_{0}^{1} \int_{0}^{+\infty} g(s) \eta_{x}^{2}(x, s) \mathrm{d} s \mathrm{~d} x \\
\leq & I_{\rho} \int_{0}^{1}(3 w-\psi)_{t}^{2} \mathrm{~d} x-\left(l-\varepsilon_{2} C_{p} G-\varepsilon_{2}\right) \int_{0}^{1}(3 w-\psi)_{x}^{2} \mathrm{~d} x+\frac{G}{4 \varepsilon_{2}} \int_{0}^{1}\left(\psi-\varphi_{x}\right)^{2} \mathrm{~d} x \\
& +\frac{g_{0}}{4 \varepsilon_{2}} \int_{0}^{1} \int_{0}^{+\infty} g(s) \eta_{x}^{2}(x, s) \mathrm{d} s \mathrm{~d} x \\
\leq & I_{\rho} \int_{0}^{1}(3 w-\psi)_{t}^{2} \mathrm{~d} x-\left(l-\varepsilon_{2}\right) \int_{0}^{1}(3 w-\psi)_{x}^{2} \mathrm{~d} x+c\left(\varepsilon_{2}\right) \int_{0}^{1}\left(\psi-\varphi_{x}\right)^{2} \mathrm{~d} x \\
& +c\left(\varepsilon_{2}\right) \int_{0}^{1} \int_{0}^{+\infty} g(s) \eta_{x}^{2}(x, s) \mathrm{d} s \mathrm{~d} x
\end{aligned}
$$

where $C_{p}$ is the poincaré constant and the Lemma is proved.

Lemma 3.4 The functional $I_{3}(t)$ satisfies, for any $\varepsilon_{3}>0$,

$$
I_{3}^{\prime}(t) \leq-\left(\frac{4 \gamma}{3}-\varepsilon_{3}\right) \int_{0}^{1} w^{2} \mathrm{~d} x-D \int_{0}^{1} w_{x}^{2} \mathrm{~d} x+c\left(\varepsilon_{3}\right) \int_{0}^{1} w_{t}^{2} \mathrm{~d} x+c\left(\varepsilon_{3}\right) \int_{0}^{1}\left(\psi-\varphi_{x}\right)^{2} \mathrm{~d} x
$$

where $c\left(\varepsilon_{3}\right)$ is a positive constant depending on $\varepsilon_{3}$. 
Proof. Differentiating $I_{3}(t)$ with respect to $t$, using the third equation of problem (2.1) and integrating by parts, we obtain

$$
I_{3}^{\prime}(t)=I_{\rho} \int_{0}^{1} w_{t}^{2} \mathrm{~d} x-\frac{4 \gamma}{3} \int_{0}^{1} w^{2} \mathrm{~d} x-D \int_{0}^{1} w_{x}^{2} \mathrm{~d} x-G \int_{0}^{1} w\left(\psi-\varphi_{x}\right) \mathrm{d} x-\frac{4 \beta}{3} \int_{0}^{1} w w_{t} \mathrm{~d} x .
$$

Making use of Young's inequality for the last two terms of this equality, we get,

$$
\begin{aligned}
I_{3}^{\prime}(t) \leq & I_{\rho} \int_{0}^{1} w_{t}^{2} \mathrm{~d} x-\frac{4 \gamma}{3} \int_{0}^{1} w^{2} \mathrm{~d} x-D \int_{0}^{1} w_{x}^{2} \mathrm{~d} x+\varepsilon_{3} G \int_{0}^{1} w^{2} \mathrm{~d} x \\
& +\frac{G}{4 \varepsilon_{3}} \int_{0}^{1}\left(\psi-\varphi_{x}\right)^{2} \mathrm{~d} x+\frac{4 \varepsilon_{3} \beta}{3} \int_{0}^{1} w^{2} \mathrm{~d} x+\frac{\beta}{3 \varepsilon_{3}} \int_{0}^{1} w_{t}^{2} \mathrm{~d} x \\
\leq & \left(I_{\rho}+\frac{\beta}{3 \varepsilon_{3}}\right) \int_{0}^{1} w_{t}^{2} \mathrm{~d} x-\left(\frac{4 \gamma}{3}-\varepsilon_{3} G-\frac{4 \varepsilon_{3} \beta}{3}\right) \int_{0}^{1} w^{2} \mathrm{~d} x-D \int_{0}^{1} w_{x}^{2} \mathrm{~d} x+\frac{G}{4 \varepsilon_{3}} \int_{0}^{1}\left(\psi-\varphi_{x}\right)^{2} \mathrm{~d} x \\
\leq & c\left(\varepsilon_{3}\right) \int_{0}^{1} w_{t}^{2} \mathrm{~d} x-\left(\frac{4 \gamma}{3}-\varepsilon_{3}\right) \int_{0}^{1} w^{2} \mathrm{~d} x-D \int_{0}^{1} w_{x}^{2} \mathrm{~d} x+c\left(\varepsilon_{3}\right) \int_{0}^{1}\left(\psi-\varphi_{x}\right)^{2} \mathrm{~d} x,
\end{aligned}
$$

which is exactly $(3.8)$.

Beyond that, to overcome the difficulty brought by infinite memory, we introduce two additional functionals:

$I_{4}(t)=\frac{D \rho}{G} \int_{0}^{1} \varphi_{t}(3 w-\psi)_{x} \mathrm{~d} x-I_{\rho} \int_{0}^{1}(3 w-\psi)_{t}\left(\psi-\varphi_{x}\right) \mathrm{d} x-\frac{\rho}{G} \int_{0}^{1} \varphi_{t} \int_{0}^{+\infty} g(s)(3 w-\psi)_{x}(t-s) \psi \mathrm{d} x$

and

$$
I_{5}(t)=-I_{\rho} \int_{0}^{1}(3 w-\psi)_{t} \int_{0}^{+\infty} g(s) \eta(x, s) \mathrm{d} s \mathrm{~d} x
$$

Lemma 3.5 The functional $I_{4}(t)$ satisfies, for any $\varepsilon_{4}>0$,

$$
I_{4}^{\prime}(t) \leq-\left(3 G-\varepsilon_{4}\right) \int_{0}^{1}\left(\psi-\varphi_{x}\right)^{2} \mathrm{~d} x+\varepsilon_{4} \int_{0}^{1}(3 w-\psi)_{t}^{2} \mathrm{~d} x+\left(\varepsilon_{4}+c\left(\varepsilon_{4}\right)\right) \int_{0}^{1} w_{t}^{2} \mathrm{~d} x+\varepsilon_{4} \int_{0}^{1} \varphi_{t}^{2} \mathrm{~d} x
$$

where $c\left(\varepsilon_{4}\right)$ is a positive constant depending on $\varepsilon_{4}$.

Proof. Taking the derivative of $I_{4}(t)$ with respect to $t$ and using the first two equations of problem 2.5, we have

$$
\begin{aligned}
I_{4}^{\prime}(t)= & -D \int_{0}^{1}\left(\psi-\varphi_{x}\right)_{x}(3 w-\psi)_{x} \mathrm{~d} x+\frac{D \rho}{G} \int_{0}^{1} \varphi_{t}(3 w-\psi)_{x t} \mathrm{~d} x-G \int_{0}^{1}\left(\psi-\varphi_{x}\right)^{2} \mathrm{~d} x \\
& -l \int_{0}^{1}(3 w-\psi)_{x x}\left(\psi-\varphi_{x}\right) \mathrm{d} x-\int_{0}^{1}\left(\psi-\varphi_{x}\right) \int_{0}^{+\infty} g(s) \eta_{x x} \mathrm{~d} s \mathrm{~d} x \\
& -I_{\rho} \int_{0}^{1}(3 w-\psi)_{t}\left(\psi-\varphi_{x}\right)_{t} \mathrm{~d} x+\int_{0}^{1}\left(\psi-\varphi_{x}\right)_{x} \int_{0}^{+\infty} g(s)(3 w-\psi)_{x}(t-s) \mathrm{d} s \mathrm{~d} x \\
& -\frac{\rho}{G} \int_{0}^{1} \varphi_{t} \int_{0}^{+\infty} g(s)(3 w-\psi)_{x t}(t-s) \mathrm{d} s \mathrm{~d} x .
\end{aligned}
$$

Using (2.3), the last two terms in 3.10 can be rewritten as follows

$$
\int_{0}^{1}\left(\psi-\varphi_{x}\right)_{x} \int_{0}^{+\infty} g(s)(3 w-\psi)_{x}(t-s) \mathrm{d} s \mathrm{~d} x-\frac{\rho}{G} \int_{0}^{1} \varphi_{t} \int_{0}^{+\infty} g(s)(3 w-\psi)_{x t}(t-s) \mathrm{d} s \mathrm{~d} x
$$




$$
\begin{aligned}
= & \int_{0}^{1}\left(\psi-\varphi_{x}\right)_{x} \int_{0}^{+\infty} g(s)(3 w-\psi)_{x} \mathrm{~d} s \mathrm{~d} x-\int_{0}^{1}\left(\psi-\varphi_{x}\right)_{x} \int_{0}^{+\infty} g(s) \eta_{x} \mathrm{~d} s \mathrm{~d} x \\
& -\frac{\rho}{G} \int_{0}^{1} \varphi_{t} \int_{0}^{+\infty} g(s)(3 w-\psi)_{x t} \mathrm{~d} s \mathrm{~d} x+\frac{\rho}{G} \int_{0}^{1} \varphi_{t} \int_{0}^{+\infty} g(s) \eta_{x t} \mathrm{~d} s \mathrm{~d} x .
\end{aligned}
$$

Then, thanks to (2.4), (3.10) and (3.11), integrating by parts, we have

$$
\begin{aligned}
I_{4}^{\prime}(t)= & -G \int_{0}^{1}\left(\psi-\varphi_{x}\right)^{2}+\left(\frac{D \rho}{G}-I_{\rho}\right) \int_{0}^{1} \varphi_{t}(3 w-\psi)_{x t} \mathrm{~d} x-I_{\rho} \int_{0}^{1}(3 w-\psi)_{t} \psi_{t} \mathrm{~d} x \\
& +\frac{\rho}{G} \int_{0}^{1} \varphi_{t} \int_{0}^{+\infty} g^{\prime}(s) \eta_{x} \mathrm{~d} s \mathrm{~d} x .
\end{aligned}
$$

Next, using Young's and Hölder's inequalities for the last two terms of this equality, we obtain

$$
\begin{aligned}
I_{4}^{\prime}(t) \leq & -G \int_{0}^{1}\left(\psi-\varphi_{x}\right)^{2} \mathrm{~d} x+\left(\frac{D \rho}{G}-I_{\rho}\right) \int_{0}^{1} \varphi_{t}(3 w-\psi)_{x t} \mathrm{~d} x+\varepsilon_{4} I_{\rho} \int_{0}^{1} \psi_{t}^{2} \mathrm{~d} x \\
& +\frac{I_{\rho}}{4 \varepsilon_{4}} \int_{0}^{1}(3 w-\psi)_{t}^{2} \mathrm{~d} x+\frac{\varepsilon_{4} \rho}{G} \int_{0}^{1} \varphi_{t}^{2} \mathrm{~d} x-\frac{\rho g(0)}{4 \varepsilon_{4} G} \int_{0}^{1} \int_{0}^{+\infty} g^{\prime}(s) \eta_{x}^{2} \mathrm{~d} s \mathrm{~d} x .
\end{aligned}
$$

It is worth noting that

$$
\int_{0}^{1} \psi_{t}^{2} \mathrm{~d} x=\int_{0}^{1}(\psi-3 w+3 w)_{t}^{2} \mathrm{~d} x
$$

Hence

$$
\begin{aligned}
I_{4}^{\prime}(t) \leq & -G \int_{0}^{1}\left(\psi-\varphi_{x}\right)^{2} \mathrm{~d} x+\left(\frac{D \rho}{G}-I_{\rho}\right) \int_{0}^{1} \varphi_{t}(3 w-\psi)_{x t} \mathrm{~d} x+2 \varepsilon_{4} I_{\rho} \int_{0}^{1}(3 w-\psi)_{t}^{2} \mathrm{~d} x \\
& +18 \varepsilon_{4} I_{\rho} \int_{0}^{1} w_{t}^{2} \mathrm{~d} x+\frac{I_{\rho}}{4 \varepsilon_{4}} \int_{0}^{1}(3 w-\psi)_{t}^{2} \mathrm{~d} x+\frac{\varepsilon_{4} \rho}{G} \int_{0}^{1} \varphi_{t}^{2} \mathrm{~d} x-\frac{\rho g(0)}{4 \varepsilon_{4} G} \int_{0}^{1} \int_{0}^{+\infty} g^{\prime}(s) \eta_{x}^{2} \mathrm{~d} s \mathrm{~d} x \\
\leq & -G \int_{0}^{1}\left(\psi-\varphi_{x}\right)^{2} \mathrm{~d} x+\left(2 \varepsilon_{4} I_{\rho}+\frac{I_{\rho}}{4 \varepsilon_{4}}\right) \int_{0}^{1}(3 w-\psi)_{t}^{2} \mathrm{~d} x+18 \varepsilon_{4} I_{\rho} \int_{0}^{1} w_{t}^{2} \mathrm{~d} x+\frac{\varepsilon_{4} \rho}{G} \int_{0}^{1} \varphi_{t}^{2} \mathrm{~d} x \\
& -\frac{\rho g(0)}{4 \varepsilon_{4} G} \int_{0}^{1} \int_{0}^{+\infty} g^{\prime}(s) \eta_{x}^{2} \mathrm{~d} s \mathrm{~d} x+\left(\frac{D \rho}{G}-I_{\rho}\right) \int_{0}^{1} \varphi_{t}(3 w-\psi)_{x t} \mathrm{~d} x \\
\leq & -G \int_{0}^{1}\left(\psi-\varphi_{x}\right)^{2} \mathrm{~d} x+\left(\varepsilon_{4}+c\left(\varepsilon_{4}\right)\right) \int_{0}^{1}(3 w-\psi)_{t}^{2} \mathrm{~d} x+\varepsilon_{4} \int_{0}^{1} \varphi_{t}^{2} \mathrm{~d} x+\varepsilon_{4} \int_{0}^{1} w_{t}^{2} \mathrm{~d} x \\
& -c\left(\varepsilon_{4}\right) \int_{0}^{1} \int_{0}^{+\infty} g^{\prime}(s) \eta_{x}^{2} \mathrm{~d} s \mathrm{~d} x+\left(\frac{D \rho}{G}-I_{\rho}\right) \int_{0}^{1} \varphi_{t}(3 w-\psi)_{x t} \mathrm{~d} x .
\end{aligned}
$$

This completes the proof of the lemma.

Lemma 3.6 The functional $I_{5}(t)$ satisfies, for any $\varepsilon_{5}>0$,

$$
\begin{aligned}
I_{5}^{\prime}(t) \leq & -\left(I_{\rho} g_{0}-\varepsilon_{5}\right) \int_{0}^{1}(3 w-\psi)_{t}^{2} \mathrm{~d} x+\varepsilon_{5} \int_{0}^{1}\left(\psi-\varphi_{x}\right)^{2} \mathrm{~d} x+\varepsilon_{5} \int_{0}^{1}(3 w-\psi)_{x}^{2} \mathrm{~d} x \\
& -c\left(\varepsilon_{5}\right) \int_{0}^{1} \int_{0}^{+\infty} g^{\prime}(s) \eta_{x}^{2} \mathrm{~d} s \mathrm{~d} x+c\left(\varepsilon_{5}\right) \int_{0}^{1} \int_{0}^{+\infty} g(s) \eta_{x}^{2} \mathrm{~d} s \mathrm{~d} x
\end{aligned}
$$

where $c\left(\varepsilon_{5}\right)$ is a positive constant depending on $\varepsilon_{5}$. 
Proof. Differentiating $I_{5}(t)$ with respect to $t$, using the second equation of problem (2.1), integrating by parts and using the fact that

$$
\begin{aligned}
\partial_{t} \int_{0}^{+\infty} g(s) \eta(s) \mathrm{d} s= & \partial_{t} \int_{0}^{+\infty} g(t-s)((3 w-\psi)(t)-(3 w-\psi)(s)) \mathrm{d} s \\
= & \int_{0}^{+\infty} g^{\prime}(t-s)((3 w-\psi)(t)-(3 w-\psi)(s)) \mathrm{d} s \\
& +\left(\int_{0}^{+\infty} g(t-s) \mathrm{d} s\right)(3 w-\psi)_{t} \\
= & \int_{0}^{+\infty} g^{\prime}(s) \eta(s) \mathrm{d} s+g_{0}(3 w-\psi)_{t},
\end{aligned}
$$

we get

$$
\begin{aligned}
I_{5}^{\prime}(t)= & -I_{\rho} g_{0} \int_{0}^{1}(3 w-\psi)_{t}^{2} \mathrm{~d} x-G \int_{0}^{1}\left(\psi-\varphi_{x}\right) \int_{0}^{+\infty} g(s) \eta(x, s) \mathrm{d} s \mathrm{~d} x \\
& +l \int_{0}^{1}(3 w-\psi)_{x} \int_{0}^{+\infty} g(s) \eta_{x}(x, s) \mathrm{d} s \mathrm{~d} x+\int_{0}^{1}\left(\int_{0}^{+\infty} g(s) \eta_{x}(x, s) \mathrm{d} s\right)^{2} \mathrm{~d} x \\
& -I_{\rho} \int_{0}^{1}(3 w-\psi)_{t} \int_{0}^{+\infty} g^{\prime}(s) \eta(x, s) \mathrm{d} s \mathrm{~d} x .
\end{aligned}
$$

Then, using Young's, Poincaré's and Hölder's inequalities for the last four terms of this equality, we deduce

$$
\begin{aligned}
I_{5}^{\prime}(t) \leq & -I_{\rho} g_{0} \int_{0}^{1}(3 w-\psi)_{t}^{2} \mathrm{~d} x+\int_{0}^{1}\left(\int_{0}^{+\infty} g(s) \eta_{x}(x, s) \mathrm{d} s\right)^{2} \mathrm{~d} x+\varepsilon_{5} G \int_{0}^{1}\left(\psi-\varphi_{x}\right)^{2} \mathrm{~d} x \\
& +\frac{C_{p} G}{4 \varepsilon_{5}} \int_{0}^{1}\left(\int_{0}^{+\infty} g(s) \eta_{x}(x, s) \mathrm{d} s\right)^{2} \mathrm{~d} x+\varepsilon_{5} l \int_{0}^{1}(3 w-\psi)_{x}^{2} \mathrm{~d} x \\
& +\frac{l}{4 \varepsilon_{5}} \int_{0}^{1}\left(\int_{0}^{+\infty} g(s) \eta_{x}(x, s) \mathrm{d} s\right)^{2} \mathrm{~d} x+\varepsilon_{5} I_{\rho} \int_{0}^{1}(3 w-\psi)_{t}^{2} \mathrm{~d} x \\
& +\frac{C_{p} I_{\rho}}{4 \varepsilon_{5}} \int_{0}^{1}\left(\int_{0}^{+\infty} g^{\prime}(s) \eta_{x} \mathrm{~d} s\right)^{2} \mathrm{~d} x \\
\leq & -I_{\rho} g_{0} \int_{0}^{1}(3 w-\psi)_{t}^{2} \mathrm{~d} x+g_{0} \int_{0}^{1} \int_{0}^{+\infty} g(s) \eta_{x}^{2}(x, s) \mathrm{d} s \mathrm{~d} x+\varepsilon_{5} G \int_{0}^{1}\left(\psi-\varphi_{x}\right)^{2} \mathrm{~d} x \\
& +\frac{C_{p} G g_{0}}{4 \varepsilon_{5}} \int_{0}^{1} \int_{0}^{+\infty} g(s) \eta_{x}^{2}(x, s) \mathrm{d} s \mathrm{~d} x+\varepsilon_{5} l \int_{0}^{1}(3 w-\psi)_{x}^{2} \mathrm{~d} x \\
& +\frac{l g_{0}}{4 \varepsilon_{5}} \int_{0}^{1} \int_{0}^{+\infty} g(s) \eta_{x}^{2}(x, s) \mathrm{d} s \mathrm{~d} x+\varepsilon_{5} I_{\rho} \int_{0}^{1}(3 w-\psi)_{t}^{2} \mathrm{~d} x \\
& -\frac{C_{p} I_{\rho} g(0)}{4 \varepsilon_{5}} \int_{0}^{1} \int_{0}^{+\infty} g^{\prime}(s) \eta_{x}^{2}(x, s) \mathrm{d} s \mathrm{~d} x \\
\leq & -\left(I_{\rho} g_{0}-\varepsilon_{5}\right) \int_{0}^{1}(3 w-\psi)_{t}^{2} \mathrm{~d} x+\varepsilon_{5} \int_{0}^{1}\left(\psi-\varphi_{x}\right)^{2} \mathrm{~d} x+\varepsilon_{5} \int_{0}^{1}(3 w-\psi)_{x}^{2} \mathrm{~d} x \\
& +c\left(\varepsilon_{5}\right) \int_{0}^{1} \int_{0}^{+\infty} g(s) \eta_{x}^{2}(x, s) \mathrm{d} s \mathrm{~d} x-c\left(\varepsilon_{5}\right) \int_{0}^{1} \int_{0}^{+\infty} g^{\prime}(s) \eta_{x}^{2}(x, s) \mathrm{d} s \mathrm{~d} x
\end{aligned}
$$

where $C_{p}$ is the poincaré constant and the proof is complete. Now, we are in a position to prove our main result. Let $\delta_{1}, \delta_{2}, \delta_{3}, \delta_{4}, \delta_{5}>0$, we define

$$
L(t)=E(t)+\delta_{1} I_{1}(t)+\delta_{2} I_{2}(t)+\delta_{3} I_{3}(t)+\delta_{4} I_{4}(t)+\delta_{5} I_{5}(t) .
$$


Using the Cauchy-Schwarz inequality and the Poincaré inequality, one can easily see that all the $I_{i}(t), i=1,2,3,4,5$ are bounded by an expression containing the existing terms in the energy $E(t)$. This leads to the equivalence of $L(t)$ and $E(t)$.

Gathering the estimates in the previous lemmas, we obtain

$$
\begin{aligned}
L^{\prime}(t) \leq & -\left(\delta_{1} \rho-\delta_{4} \varepsilon_{4}\right) \int_{0}^{1} \varphi_{t}^{2} \mathrm{~d} x-\left(\delta_{5} I_{\rho} g_{0}-\delta_{2} I_{\rho}-\delta_{4} \varepsilon_{4}-\delta_{4} c\left(\varepsilon_{4}\right)-\delta_{5} \varepsilon_{5}\right) \int_{0}^{1}(3 w-\psi)_{t}^{2} \mathrm{~d} x \\
& -\left(4 \beta-\delta_{3} c\left(\varepsilon_{3}\right)-\delta_{4} \varepsilon_{4}\right) \int_{0}^{1} w_{t}^{2} \mathrm{~d} x-\left(\delta_{3} D-\delta_{1} c\left(\varepsilon_{1}\right)\right) \int_{0}^{1} w_{x}^{2} \mathrm{~d} x \\
& -\left(\delta_{4} G-\delta_{1} G-\delta_{1} \varepsilon_{1}-\delta_{2} c\left(\varepsilon_{2}\right)-\delta_{3} c\left(\varepsilon_{3}\right)-\delta_{5} \varepsilon_{5}\right) \int_{0}^{1}\left(\psi-\varphi_{x}\right)^{2} \mathrm{~d} x \\
& -\left(\delta_{2} l-\delta_{1} c\left(\varepsilon_{1}\right)-\delta_{2} \varepsilon_{2}-\delta_{5} \varepsilon_{5}\right) \int_{0}^{1}(3 w-\psi)_{x}^{2} \mathrm{~d} x-\left(\frac{4 \gamma}{3} \delta_{3}-\delta_{3} \varepsilon_{3}\right) \int_{0}^{1} w^{2} \mathrm{~d} x \\
& +\left(\frac{1}{2}-\delta_{4} c\left(\varepsilon_{4}\right)-\delta_{5} c\left(\varepsilon_{5}\right)\right) \int_{0}^{1} \int_{0}^{+\infty} g^{\prime}(s) \eta_{x}^{2} \mathrm{~d} s \mathrm{~d} x \\
& +\left(\delta_{2} c\left(\varepsilon_{2}\right)+\delta_{5} c\left(\varepsilon_{5}\right)\right) \int_{0}^{1} \int_{0}^{+\infty} g(s) \eta_{x}^{2} \mathrm{~d} s \mathrm{~d} x+\left(\frac{D \rho}{G}-I_{\rho}\right) \int_{0}^{1} \varphi_{t}(3 w-\psi)_{x t} \mathrm{~d} x .
\end{aligned}
$$

At this point, we need to choose our constants very carefully. First, we choose $\varepsilon_{1}, \varepsilon_{2}, \varepsilon_{3}, \varepsilon_{4}, \varepsilon_{5}$ small enough so that

$$
\begin{aligned}
L^{\prime}(t) \leq & -\frac{\delta_{1} \rho}{2} \int_{0}^{1} \varphi_{t}^{2} \mathrm{~d} x-\left(\frac{\delta_{5} I_{\rho} g_{0}}{2}-\delta_{2} I_{\rho}-\delta_{4} c\left(\varepsilon_{4}\right)\right) \int_{0}^{1}\left(3 w_{t}-\psi_{t}\right)^{2} \mathrm{~d} x-\left(2 \beta-\delta_{3} c\left(\varepsilon_{3}\right)\right) \int_{0}^{1} w_{t}^{2} \mathrm{~d} x \\
& -\left(\delta_{3} D-\delta_{1} c\left(\varepsilon_{1}\right)\right) \int_{0}^{1} w_{x}^{2} \mathrm{~d}-\left(\frac{\delta_{4} G}{2}-\delta_{1} G-\delta_{2} c\left(\varepsilon_{2}\right)-\delta_{3} c\left(\varepsilon_{3}\right)\right) \int_{0}^{1}\left(\psi-\varphi_{x}\right)^{2} \mathrm{~d} x \\
& -\left(\frac{\delta_{2} l}{2}-\delta_{1} c\left(\varepsilon_{1}\right)\right) \int_{0}^{1}\left(3 w_{x}-\psi_{x}\right)^{2} \mathrm{~d} x-\frac{2 \delta_{3} \gamma}{3} \int_{0}^{1} w^{2} \mathrm{~d} x \\
& +\left(\frac{1}{2}-\delta_{4} c\left(\varepsilon_{4}\right)-\delta_{5} c\left(\varepsilon_{5}\right)\right) \int_{0}^{1} \int_{0}^{+\infty} g^{\prime}(s) \eta_{x}^{2} \mathrm{~d} s \mathrm{~d} x \\
& +\left(\delta_{2} c\left(\varepsilon_{2}\right)+\delta_{5} c\left(\varepsilon_{5}\right)\right) \int_{0}^{1} \int_{0}^{+\infty} g(s) \eta_{x}^{2} \mathrm{~d} s \mathrm{~d} x+\left(\frac{D \rho}{G}-I_{\rho}\right) \int_{0}^{1} \varphi_{t}(3 w-\psi)_{x t} \mathrm{~d} x .
\end{aligned}
$$

Second, we select $\delta_{5}$ small enough so that

$$
\frac{1}{2}-\delta_{5} c\left(\varepsilon_{5}\right)>0
$$

Third, we choose $\delta_{4}$ small enough so that

$$
\frac{\delta_{5} I_{\rho} g_{0}}{2}-\delta_{4} c\left(\varepsilon_{4}\right)>0 \text { and } \quad \frac{1}{2}-\delta_{4} c\left(\varepsilon_{4}\right)-\delta_{5} c\left(\varepsilon_{5}\right)>0 .
$$

Then, we select $\delta_{3}$ small enough so that

$$
2 \beta-\delta_{3} c\left(\varepsilon_{3}\right)>0 \text { and } \quad \frac{\delta_{4} G}{2}-\delta_{3} c\left(\varepsilon_{3}\right)>0 .
$$

Next, we choose $\delta_{2}$ small enough so that

$$
\frac{\delta_{5} I_{\rho} g_{0}}{2}-\delta_{2} I_{\rho}-\delta_{4} c\left(\varepsilon_{4}\right)>0 \quad \text { and } \quad \frac{\delta_{4} G}{2}-\delta_{2} c\left(\varepsilon_{2}\right)-\delta_{3} c\left(\varepsilon_{3}\right)>0 .
$$


Finally, we select $\delta_{1}$ small enough so that

$$
\delta_{3} D-\delta_{1} c\left(\varepsilon_{1}\right)>0, \quad \frac{3 \delta_{4} G}{2}-\delta_{1} G-\delta_{2} c\left(\varepsilon_{2}\right)-\delta_{3} c\left(\varepsilon_{3}\right)>0 \quad \text { and } \quad \frac{\delta_{2} l}{2}-\delta_{1} c\left(\varepsilon_{1}\right)>0 .
$$

From the above, we deduce that there exist positive constants $C_{1}$ and $C_{2}$ such that $(3.16)$ becomes

$$
L^{\prime}(t) \leq-C_{1} E(t)+C_{2} \int_{0}^{1} \int_{0}^{+\infty} g(s) \eta_{x}^{2}(x, s) \mathrm{d} s \mathrm{~d} x+\left(\frac{D \rho}{G}-I_{\rho}\right) \int_{0}^{1} \varphi_{t}(3 w-\psi)_{x t} \mathrm{~d} x .
$$

Now, we estimate the second term in the right hand side of (3.17). This is the main difficulty in the treating of the infinite memories. To overcome this difficulty, we have the following lemma:

Lemma 3.7 For any $\epsilon_{0}>0$, there exist positive constants c such that, we have the following inequalities:

$$
G^{\prime}\left(\epsilon_{0} E(t)\right) \int_{0}^{L} \int_{0}^{+\infty} g(s) \eta_{x}^{2} \mathrm{~d} s \mathrm{~d} x \leq-c E^{\prime}(t)+c \epsilon_{0} E(t) G^{\prime}\left(\epsilon_{0} E(t)\right) .
$$

Proof. The proof of this lemma is similar to the proof of Lemma 3.11 in [6] and is omitted.

Now, going back to the proof of Theorem 2.1, multiplying (3.17) by $G^{\prime}\left(\epsilon_{0} E(t)\right)$, using (3.18), we obtain

$$
\begin{aligned}
G^{\prime}\left(\epsilon_{0} E(t)\right) L^{\prime}(t) \leq & -\left(c-c \epsilon_{0}\right) E(t) G^{\prime}\left(\epsilon_{0} E(t)\right)-c E^{\prime}(t) \\
& +\left(\frac{D \rho}{G}-I_{\rho}\right) G^{\prime}\left(\epsilon_{0} E(t)\right) \int_{0}^{1} \varphi_{t}(3 w-\psi)_{x t} \mathrm{~d} x .
\end{aligned}
$$

Choosing $\epsilon_{0}$ small enough, we have

$$
G^{\prime}\left(\epsilon_{0} E(t)\right) L^{\prime}(t)+c E^{\prime}(t) \leq-c E(t) G^{\prime}\left(\epsilon_{0} E(t)\right)+\left(\frac{D \rho}{G}-I_{\rho}\right) G^{\prime}\left(\epsilon_{0} E(t)\right) \int_{0}^{1} \varphi_{t}(3 w-\psi)_{x t} \mathrm{~d} x
$$

Let

$$
F(t)=G^{\prime}\left(\epsilon_{0} E(t)\right) L(t)+c E(t) \sim E(t),
$$

because $L(t) \sim E(t)$ and $G^{\prime}\left(\epsilon_{0} E(t)\right)$ is non-increasing. Using (3.20), we have

$$
F^{\prime}(t) \leq-c E(t) G^{\prime}\left(\epsilon_{0} E(t)\right)+\left(\frac{D \rho}{G}-I_{\rho}\right) G^{\prime}\left(\epsilon_{0} E(t)\right) \int_{0}^{1} \varphi_{t}(3 w-\psi)_{x t} \mathrm{~d} x .
$$

Next, to estimate the last term of (3.21), we define the second-order energy by

$$
\tilde{E}(t)=E\left(U_{t}(t)\right)
$$

where $E(U(t))=E(t)(E(t)$ is defined by 2.9) ). A simple calculation (as for (3.1)) implies that

$$
\tilde{E}^{\prime}(t)=-4 \beta \int_{0}^{L} w_{t t} \mathrm{~d} x+\frac{1}{2} \int_{0}^{L} \int_{0}^{+\infty} g^{\prime}(s) \eta_{x t}^{2} \mathrm{~d} s \mathrm{~d} x \leq 0 .
$$

Now, we proceed as in [6] to establish the following lemma. 
Lemma 3.8 For any $\epsilon>0$, we have the following inequalities:

$$
\begin{aligned}
\left(\frac{D \rho}{G}-I_{\rho}\right) \int_{0}^{1} \varphi_{t}(3 w-\psi)_{x t} \mathrm{~d} x \leq & \epsilon E(t) \\
& +c(\epsilon)\left(\int_{0}^{L} \int_{0}^{+\infty} g(s) \eta_{x t}^{2} \mathrm{~d} s \mathrm{~d} x-\int_{0}^{L} \int_{0}^{+\infty} g^{\prime}(s) \eta_{x}^{2} \mathrm{~d} s \mathrm{~d} x\right) .
\end{aligned}
$$

Proof. By recalling that $g_{0}=\int_{0}^{+\infty} g(s) \mathrm{d} s$, we have

$$
\begin{aligned}
\left(\frac{D \rho}{G}-I_{\rho}\right) \int_{0}^{1} \varphi_{t}(3 w-\psi)_{x t} \mathrm{~d} x= & \frac{\frac{D \rho}{G}-I_{\rho}}{g_{0}} \int_{0}^{1} \varphi_{t} \int_{0}^{+\infty} g(s) \eta_{x t} \mathrm{~d} s \mathrm{~d} x \\
& +\frac{\frac{D \rho}{G}-I_{\rho}}{g_{0}} \int_{0}^{1} \varphi_{t} \int_{0}^{+\infty} g(s)(3 w-\psi)_{x t}(t-s) \mathrm{d} s \mathrm{~d} x .
\end{aligned}
$$

Using Young's and Hölder's inequalities, we obtain, for all $\epsilon>0$,

$$
\begin{aligned}
\frac{\frac{D \rho}{G}-I_{\rho}}{g_{0}} \int_{0}^{1} \varphi_{t} \int_{0}^{+\infty} g(s) \eta_{x t} \mathrm{~d} s \mathrm{~d} x & \leq c \int_{0}^{1}\left|\varphi_{t}\right| \int_{0}^{+\infty} g(s)\left|\eta_{x t}\right| \mathrm{d} s \mathrm{~d} x \\
& \leq c \epsilon \int_{0}^{1} \varphi_{t}^{2} \mathrm{~d} x+\frac{c}{4 \epsilon} \int_{0}^{1}\left(\int_{0}^{+\infty} g(s) \eta_{x t} \mathrm{~d} s\right)^{2} \mathrm{~d} x \\
& \leq c \epsilon \int_{0}^{1} \varphi_{t}^{2} \mathrm{~d} x+\frac{c g_{0}}{4 \epsilon} \int_{0}^{1} \int_{0}^{+\infty} g(s) \eta_{x t}^{2} \mathrm{~d} s \mathrm{~d} x \\
& \leq \frac{\epsilon}{2} E(t)+c(\epsilon) \int_{0}^{1} \int_{0}^{+\infty} g(s) \eta_{x t}^{2} \mathrm{~d} s \mathrm{~d} x
\end{aligned}
$$

On the other hand, integrating by parts, exploiting (2.3), (2.4) and using Young's and Hölder's inequalities, we get

$$
\begin{aligned}
\frac{\frac{D \rho}{G}-I_{\rho}}{g_{0}} \int_{0}^{1} \varphi_{t} \int_{0}^{+\infty} g(s)(3 w-\psi)_{x t}(t-s) \mathrm{d} s \mathrm{~d} x & =\frac{\frac{D \rho}{G}-I_{\rho}}{g_{0}} \int_{0}^{1} \varphi_{t} \int_{0}^{+\infty} g(s)\left(\eta_{x t}+\eta_{x s}-\eta_{x t}\right) \mathrm{d} s \mathrm{~d} x \\
& =\frac{\frac{D \rho}{G}-I_{\rho}}{g_{0}} \int_{0}^{1} \varphi_{t} \int_{0}^{+\infty}-g^{\prime}(s) \eta_{x} \mathrm{~d} s \mathrm{~d} x \\
& \leq \frac{\epsilon}{2} E(t)-c(\epsilon) \int_{0}^{1} \int_{0}^{+\infty} g^{\prime}(s) \eta_{x}^{2} \mathrm{~d} s \mathrm{~d} x .
\end{aligned}
$$

Finally, adding above two equations, the proof is completed.

Now, going back to the proof of Theorem 2.1, using (3.21) and choosing $\epsilon$ small enough, we obtain

$$
F^{\prime}(t) \leq-c E(t) G^{\prime}\left(\epsilon_{0} E(t)\right)+c G^{\prime}\left(\epsilon_{0} E(t)\right)\left(\int_{0}^{L} \int_{0}^{+\infty} g(s) \eta_{x t}^{2} \mathrm{~d} s \mathrm{~d} x-\int_{0}^{L} \int_{0}^{+\infty} g^{\prime}(s) \eta_{x}^{2} \mathrm{~d} s \mathrm{~d} x\right)
$$

Using (3.1) and the fact that $G^{\prime}\left(\epsilon_{0} E(t)\right)$ is non-increasing, we have

$$
E(t) G^{\prime}\left(\epsilon_{0} E(t)\right) \leq-c G^{\prime}\left(\epsilon_{0} E(0)\right) E^{\prime}(t)-c F^{\prime}(t)+c G^{\prime}\left(\epsilon_{0} E(t)\right) \int_{0}^{L} \int_{0}^{+\infty} g(s) \eta_{x t}^{2} \mathrm{~d} s \mathrm{~d} x .
$$


Next, we estimate the last term in 3.25 . Similarly to the case of $\int_{0}^{L} \int_{0}^{+\infty} g(s) \eta_{x t}^{2} \mathrm{~d} s \mathrm{~d} x$ in Lemma 3.7 (for $\int_{0}^{L} \int_{0}^{+\infty} g(s) \eta_{x t}^{2} \mathrm{~d} s \mathrm{~d} x$ instead of $\int_{0}^{L} \int_{0}^{+\infty} g(s) \eta_{x}^{2} \mathrm{~d} s \mathrm{~d} x$, we get, using (2.10) and 3.22,

$$
G^{\prime}\left(\epsilon_{0} E(t)\right) \int_{0}^{L} \int_{0}^{+\infty} g(s) \eta_{x t}^{2} \mathrm{~d} s \mathrm{~d} x \leq-c \tilde{E}^{\prime}(t)+c \epsilon_{0} E(t) G^{\prime}\left(\epsilon_{0} E(t)\right) .
$$

Then, (3.25) and $(3.26)$ with $\epsilon_{0}$ chosen small enough imply that

$$
E(t) G^{\prime}\left(\epsilon_{0} E(t)\right) \leq-c G^{\prime}\left(\epsilon_{0} E(0)\right) E^{\prime}(t)-c F^{\prime}(t)-c \tilde{E}^{\prime}(t) .
$$

Recalling the fact that $F(t) \sim E(t)$ and $E(t) G^{\prime}\left(\epsilon_{0} E(t)\right)$ is non-increasing, we arrive at, for all $T \in \mathbb{R}_{+}\left(G_{0}\right.$ is defined by $\left.(2.12)\right)$,

$$
G_{0}(E(T)) T \leq \int_{0}^{T} G_{0}(E(t)) \mathrm{d} t \leq c\left(G^{\prime}\left(\epsilon_{0} E(0)\right)+1\right) E(0)+c \tilde{E}(0),
$$

which gives 2.11) with $C=c\left(G^{\prime}\left(\epsilon_{0} E(0)\right)+1\right) E(0)+c \tilde{E}(0)$. This completes the proof of Theorem 2.1 .

\section{References}

[1] H. Brezis, Functional analysis, Sobolev spaces and partial differential equations, Universitext, Springer, New York, 2011.

[2] C. M. Dafermos, Asymptotic stability in viscoelasticity, Arch. Rational Mech. Anal. 37 (1970), 297-308.

[3] M. Ferhat and A. Hakem, Well-posedness and asymptotic stability of solutions to a Bresse system with time varying delay terms and infinite memories, Facta Univ. Ser. Math. Inform. 31 (2016), no. 1, 97-124.

[4] A. Guesmia, On the stabilization for Timoshenko system with past history and frictional damping controls, Palest. J. Math. 2 (2013), no. 2, 187-214.

[5] A. Guesmia and S. Messaoudi, Some stability results for Timoshenko systems with cooperative frictional and infinite-memory dampings in the displacement, Acta Math. Sci. Ser. B Engl. Ed. 36 (2016), no. 1, 1-33.

[6] A. Guesmia, S. A. Messaoudi and A. Soufyane, Stabilization of a linear Timoshenko system with infinite history and applications to the Timoshenko-heat systems, Electron. J. Differential Equations 2012, No. 193, 45 pp.

[7] S. W. Hansen and R. Spies, Structural damping in a laminated beams due to interfacial slip, J. Sound Vibration. 204 (1997), no. 2, 183-202.

[8] J.-R. Kang, Long-time behavior of a suspension bridge equations with past history, Appl. Math. Comput. 265 (2015), 509-519.

[9] V. Komornik, Exact controllability and stabilization, RAM: Research in Applied Mathematics, Masson, Paris, 1994. 
[10] A. Lo and N. Tatar, Stabilization of laminated beams with interfacial slip, Electron. J. Differential Equations 2015, No. 129, 14 pp.

[11] A. Lo and N. Tatar, Uniform stability of a laminated beam with structural memory, Qual. Theory Dyn. Syst., in press, doi: 10.1007/s12346-015-0147-y.

[12] J. E. Muñoz Rivera and H. D. Fernández Sare, Stability of Timoshenko systems with past history, J. Math. Anal. Appl. 339 (2008), no. 1, 482-502.

[13] A. Pazy, Semigroups of linear operators and applications to partial differential equations, Applied Mathematical Sciences, 44, Springer, New York, 1983.

[14] C. A. Raposo, Exponential stability for a structure with interfacial slip and frictional damping, Appl. Math. Lett. 53 (2016), 85-91.

[15] F. Tahamtani and M. Shahrouzi, Existence and blow up of solutions to a Petrovsky equation with memory and nonlinear source term, Bound. Value Probl. 2012, No. 1, 15 pp.

[16] J.-M. Wang, G.-Q. Xu and S.-P. Yung, Exponential stabilization of laminated beams with structural damping and boundary feedback controls, SIAM J. Control Optim. 44 (2005), no. $5,1575-1597$.

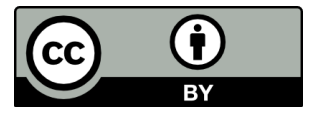

(C) 2017 by the authors; licensee Preprints, Basel, Switzerland. This article is an open access article distributed under the terms and conditions of the Creative Commons Attribution (CC BY) license http://creativecommons.org/licenses/by/4.0/). 